\section{Leonor Rosales Rojas ${ }^{1}$ Carlos Larrucea Verdugo ${ }^{2}$ Ramiro Castro Barahona ${ }^{3}$ Ariel Acevedo Avila ${ }^{4}$ Marjorie Leiva Ledea ${ }^{4}$}

${ }^{1}$ Cirujano-Dentista. Diplomada en restauraciones estéticas adhesivas.

${ }^{2}$ Profesor Asistente.

${ }^{3}$ Profesor Conferenciante.

${ }^{4}$ Docente.

Departamento de Rehabilitación Bucomaxilofacial. Área de Fisiología. Facultad de Ciencias de la Salud. Universidad de Talca. Chile.

\title{
Recidiva del grado de clareamiento dental por té: in vitro
}

Rosales-Rojas L, Larrucea-Verdugo C, Castro-Barahona R, Acevedo-Avila A, Leiva-Ledea M. Recidiva del grado de clareamiento dental por té: in vitro. Rev Estomatol Herediana. 2010; 20(2):69-77.

\section{RESUMEN}

INTRODUCCION: El referente estético en nuestra sociedad viene determinado por varios factores, el que más insatisfacción produce es un color anómalo del diente, por eso que al mejorar su color dental, los pacientes esperan buenos resultados y duraderos. OBJETIVO: Determinar la recidiva de color experimentado por piezas dentarias clareadas y posteriormente sumergidas en solución de té. METODOLOGIA: Utilizamos 42 incisivos centrales sanos de bovinos de 3 y 4 años, preparados en probetas cilíndricas acrílicas, mantenidos en saliva artificial a $37^{\circ} \mathrm{C}$, a $100 \%$ de humedad, divididas en: grupo experimental (GC), tratado con peróxido de carbamida al 35\% gel por 30 minutos y Grupo Control (GNC) no tratado. A ambos medimos color inicial con espectrofómetro Vita Easyshade ${ }^{\circledR}$ y sumergieron en solución de Té por 10 minutos. Medimos el nuevo color, realizando este procedimiento 50 veces. RESULTADOS: Analizados con, Pruebas T y Análisis de Regresión Lineal, tanto el GC como el GNC presentan el primer cambio de color con la primera inmersión en té. El segundo cambio para el GE $(p<0,029)$ es en la segunda inmersión y para el GC entre la tercera y cuarta. El cambio de color máximo fue alcanzado por el GNC entre las 20 a 30 inmersiones, sin embargo, el GC lo hace entre la 10 y 20 inmersión. CONCLUSIONES: Las piezas clareadas presentan mayor regresión del color que las piezas no tratadas. Además un color inicial claro determina cambios de más intensidad en menor tiempo y sin un patrón definido de progresión.

Palabras clave: BLANQUEAMIENTO DE DIENTES / DECOLORACIÓN DE DIENTES / TÉ.

Relapse of dental bleaching after the consumption of tea: in vitro study ABSTRACT

INTRODUCTION: Esthetic model in our society comes determined by several factors, one of the more important dissatisfaction is an anomalous color of the toothfor that reasonpatients are wating for good and long lasting results when improving the color of their teeth. OBJETIVE: To determine the color regression experienced by bleached teeth that were later plunged in a solution of tea. METHODOLOGY: 42 central permanent healthy incisor teeth from 3-4 year old bovine, supported in cylindrical acrylic and maintain in artificial saliva at $37^{\circ} \mathrm{C}$ for $100 \%$ of humidity, were divided in: experimental group (CG), treated with Carbamide Peroxide $35 \%$ gel for30 minutes and Control Group (NCG) not treated. In both, the initial color was measured with Vita Easyshade ${ }^{\circledR}$ Instruments and then plunged into Tea solution for 10 minutes. The new color was measured, and the procedure was repeated50 times. RESULTS: Analyzed with, T tests and Analysis of Lineal Regression, both the GG and the NCG present the first change of color with the first dip in Tea. The second change for the CG $(p<0.029)$ is in the second dip and for the CG between the third and fourth dip. The change of maximum color was reached by the NCG between 20 to 30 dips, nevertheless, the CG dip does it between the 10 to 20 immersions. CONCLUSIONS: Bleached teeth present the highest returnregression of the color that the not treated. In addition an initial clear color determines changes of more intensity in less time and without a definite form of progression.

Key words: TOOTH BLEACHING / TOOTH DISCOLORATION / TEA.

\section{Introducción}

Hoy son muchos los pacientes que se interesan por tratamientos odontológicos estéticos por razones diversas: desde la búsqueda de satisfacción personal hasta necesidades de tipo laboral. El referente estético dental en nuestra sociedad viene determinado por varios factores, de entre ellos, uno de los que más insatisfacción produce es un color anómalo del diente o que no satisfaga las expectativas personales. Es por ello que al intentar mejorar su color dental, los pacientes esperan muy buenos resultados, y que estos sean duraderos, de modo que el no cumplimiento de este objetivo llevará al fracaso de la adhesión del paciente al tratamiento $(1,2)$.

Los dientes son policromáticos, y su color, definido como la percepción visual de una onda de energía electromagnética de longitud determinada, comprende tres fenómenos: físico (que es la luz), psicofísico (respuesta del ojo al estímulo de la luz) y psicosensorial (que corresponde a la respuesta cerebral a los mensajes codificados enviados por los fotorreceptores del ojo) $(3,4)$.

El color de las piezas dentarias está determinado por las tres dimensiones de éste:

- Hue, Es la cualidad que distingue entre las familias de color. Corresponde a la tonalidad dada por una longitud de onda reflejada (1).

- Valor, Es la cualidad por la cual se distingue un color claro de uno oscuro es decir, corresponde a la luminosidad o brillo, siendo independiente de la tonalidad y es considerado como el determinante más importante del color (4).

- Chroma, que corresponde a la intensidad del color. En otras palabras, es la saturación de la 
tonalidad. Este chroma aumenta al aumentar la luminosidad (4).

Además, el color de los dientes está influenciado por una combinación de su color intrínseco y la presencia de cualquier mancha extrínseca que se pueda formar en la superficie de éste $(3,5)$.

La decoloración de las piezas dentarias se ha clasificado según la localización de la mancha, en intrínseca o extrínseca. También existe una denominación de decoloración internalizada que es la incorporación de la mancha extrínseca dentro de la sustancia del diente, que ocurre en defectos del esmalte y en la superficie porosa del esmalte dental expuesto (6). La etiología es multifactorial, de su formación van a ser responsables restos alimentarios, el metabolismo de distintas bacterias, la saliva, hábitos exógenos o productos de origen sérico, las pigmentaciones de taninos por té y café, compuestos de polifenoles, tales como catequinas y leucocianinas (3).

El clareamiento Dental se presenta como un procedimiento terapéutico que posibilita la eliminación de las decoloraciones dentales y proporciona, de este modo, un color adecuado en consonancia con las demandas estéticas del paciente $(7,8)$. Puede ser realizado de forma aislada o como técnica complementaria formando parte de un tratamiento dental estético global $(1,2,8)$.

Existen varias sustancias utilizadas para realizar este procedimiento, en dientes vitales, se utilizan concentraciones entre el 10 , 15, 20 y 35\%. Son soluciones muy inestables y se descomponen en peróxido de hidrógeno y urea. A su vez, el peróxido de hidrógeno se descompone en oxígeno y agua, la urea, en amonio y dióxido de carbono
$(3,9)$. Todos estos agentes clareadores, ocasionan alteraciones en la microdureza y morfología de la superficie del esmalte, existiendo una relación directa entre alteración de la superficie del esmalte y tiempo de exposición y de concentración del agente utilizado. Pero, éstas podrían ser solucionadas al aplicar soluciones de flúor posterior a este tratamiento $(10,11)$. La mayoría de los estudios in vitro, han sido realizados utilizando dientes de bovinos, los cuales se caracterizan por ser muy similares en su composición al esmalte humano, este, tiene una densidad y una dureza Vickers levemente más baja que el de bovino. $\mathrm{Al}$ someter a clareamiento a dientes de bovino se observó una disminución en el módulo elástico de dichas piezas. Sin embargo, al aplicarles posteriormente una crema dental con fluoruros se invirtió este efecto (8).

Con respecto a esto, los cambios ocasionados por lo agentes clareadores sobre las piezas dentarias, como la disolución de las capas superficiales del esmalte orgánico (película adquirida), aumento de la rugosidad superficial y de la pérdida de la capa aprismática del esmalte después de la exposición se podrían explicar principalmente por el oxígeno naciente (12). También debemos evaluar los efectos sobre la superficie dentaria de los agentes activos tras el clareamiento dental, y determinar si una vez que el diente es clareado este se vuelve más susceptible a pigmentarse nuevamente. Con respecto a esto, se concluye que después del clareamiento vital, la susceptibilidad del esmalte para pigmentarse aumenta perceptiblemente (13). En un estudio realizado posterior al clareamiento, no se observó ninguna diferencia significativa, al aplicar té directamente sobre dichas piezas dentarias, independiente del intervalo de tiempo transcurrido entre el procedimiento clareador y el contacto de la superficie del diente con té (8). Al hablar respecto de la necesidad de retratamiento, se ha observado que una vez terminado el clareamiento dental una ligera regresión del color ocurre a las dos semanas siguientes (3).

La efectividad del clareamiento dental depende de varios factores, parece que el factor que determina el éxito de la técnica es el color inicial de los dientes afectados, de modo que cuanto más clara sea la tinción, tanto más fácil resulta su clareamiento (1). La estabilidad del color puede esperarse en hasta un $90 \%$ de los pacientes después de un año del tratamiento, un $62 \%$ a tres años y al menos 35\% a los siete años. Sin embargo, en otros estudios, se ha observado que el cambio en la coloración comienza alrededor de los primeros seis meses de realizado el procedimiento (14). En un estudio, 30 pacientes fueron sometidos a clareamiento usando peróxido de carbamida al 10\%, el cual reveló que, el $43 \%$ de ellos percibieron que el color de los dientes se mantuvo estable 10 años después de dicho tratamiento (15). Sin embargo, después de la revisión bibliográfica consultada no se señala con claridad si ocurre o no recidiva del color después del clareamiento ni el periodo de tiempo en el que se manifiesta dicho fenómeno. Tampoco, se ha evaluado la verdadera influencia de ciertos hábitos como el consumo de té, café, vino, mala higiene, etc en este cambio de coloración.

En relación a este tema, no existen evidencias concretas en torno a la regresión del color experimentado por piezas dentarias 
sometidas a clareamiento, ni el cómo ocurren; también se desconoce la predictibilidad exacta de este procedimiento, es por esto que, surge la interrogante de que si el clareamiento dental tendría alguna influencia en la recidiva del color experimentados por éstas piezas, es decir, si al realizar estos procedimientos clínicos las piezas dentarias quedan más susceptibles de tener cambios de coloración (16).

Por lo tanto es razonable plantear que "en piezas clareadas la regresión del color ocurre con mayor magnitud que en aquellas no tratadas", para esto el presente estudio pretende, determinar, in vitro, la recidiva de color experimentado por piezas dentarias sometidas a clareamiento, y posteriormente sumergidas en una solución de té.

\section{Material y métodos}

Para realizar este estudio in vitro se seleccionaron 42 incisivos centrales permanentes sanos de bovinos de entre 3 y 4 años. Los cuales inmediatamente después de ser extraídos fueron conservados en una solución de cloruro de sodio al $0,9 \%$, posteriormente sumergidos en saliva artificial y mantenido a una temperatura de $37^{\circ} \mathrm{C}$ y $100 \%$ de humedad en una estufa Memment hasta el momento de comenzar el procedimiento experimental.

Las piezas dentarias fueron tratadas, procedimiento que consistió en limpiar la superficie de los dientes de restos orgánicos con una copa de goma para contrangulo y una mezcla de agua con piedra pómez de grano medio. Seguido a esto, se cortó la porción radicular con discos de carburundum dejando sólo tres milímetros de ella y posteriormente fueron vaciados y lavados los restos de contenido pulpar-cameral.

Los especimenes fueron

introducidos en cilindros de resina acrílica de autocurado rosado, de modo que la superficie vestibular quedara libre de ésta, para formar probetas cilíndricas de $4 \mathrm{~cm}$. de diámetro por tres centímetros de alto (Fig. 1).

Se midió el color de todos los especimenes de forma objetiva a través de un espectrofotómetro Vita EasyShade ${ }^{\circledR}$, basado en la guía de color Vita 3D-Master y VITAPAN (Fig. 2).

La totalidad de las probetas, se mantuvieron en las condiciones de temperatura y humedad mencionadas anteriormente durante toda la etapa experimental, y sólo fueron retiradas para realizar el procedimiento de clareamiento y posteriormente cambiadas a la infusión de té negro.

Los 42 especimenes fueron distribuidos al azar en dos grupos de igual número. Grupo 1 (GC): piezas dentarias sometidas a clareamiento (experimental). Grupo 2 (GNC): Piezas dentarias no sometidas a clareamiento (control).

Los ejemplares del Grupo Experimental (GC) fueron sometidos a clareamiento a través de la técnica de clareamiento externo con peróxido de carbamida al 35\% en gel durante 30 minutos a una temperatura de $37^{\circ} \mathrm{C}$ en una atmósfera húmeda, posteriormente fueron lavadas con agua y devueltas

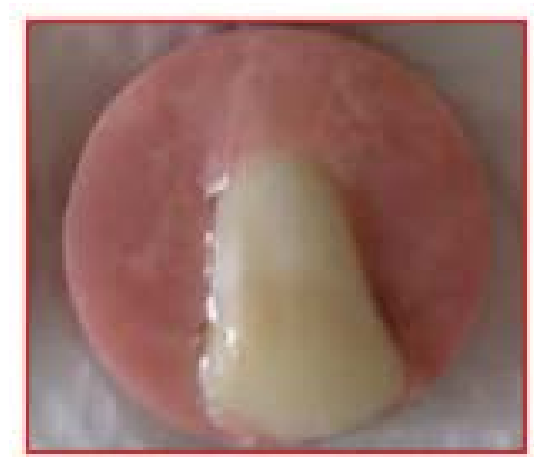

Fig. 1. Probetas de resina acrílica de autocurado. a la saliva artificial (la cual es recambiada diariamente). Después de lo cual se midió inmediatamente el color resultante de los especimenes.

La infusión de té negro fue preparada a partir de dos bolsas de té "Club" de 2 gramos cada una en $500 \mathrm{ml}$ de agua a $100^{\circ} \mathrm{C}$, dejando un periodo de 3 minutos de inmersión de dichas bolsas. Posteriormente, a una temperatura de $64^{\circ} \mathrm{C}$ (promedio de consumo), tanto los especimenes del grupo control como los del grupo experimental fueron removidos desde la saliva artificial, y sumergidos en ésta solución de té por 10 minutos. Luego, los especimenes tanto Control como Experimental, fueron lavados con agua y se realizó la medición del color en el centro de la cara vestibular de cada pieza dentaria a través del Espectrofotómetro Vita EasyShade®. Después de ello fueron devueltos a la saliva artificial, y luego de una hora, sometidos nuevamente en una nueva solución de té, en total se realizaron 50 inmersiones.

Los resultados obtenidos fueron evaluados mediante una comparación de medianas para poder establecer conclusiones de ambos grupos independientes utilizando pruebas t para muestras independientes con un 95\% de confianza.

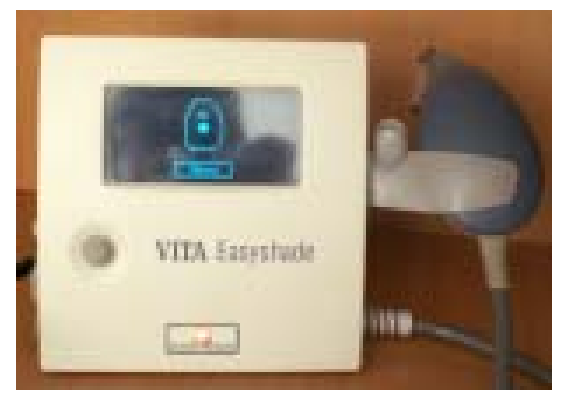

Fig. 2. Espectofotómetro Vita EasyShade. 


\section{Resultados}

Para poder analizar estadísticamente los resultados obtenidos de la etapa experimental, se realizó una equivalencia de la Guía de Color Vita 3D- Master, en la cual a cada posibilidad de color se le otorgó un número arábico, de manera que se obtuvieron 105 posibilidades de combinaciones (Tabla 1). en el GNC (Tabla 3). Como el valor de $\mathrm{p}<0,05(0,029)$ de significancia es posible afirmar que los datos son estadísticamente significativos para mencionar que las medianas del GC y del GNC difieren entre si. Esto nos permite concluir que el segundo cambio de color (Valor) ocurrió en distintas inmersiones, siendo para el GC en su mayoría, durante la segunda inmersión en té y para el GNC entre la tercera y cuarta inmersión (Fig. 4).

3.En qué número de inmersión en té ocurre el cambio de coloración máximo (5M3=105) en el GC y el GNC (Tabla 4). Como el valor de $\mathrm{p}>0,05(0,272)$, entonces con un 95\% de significancia es posible afirmar que la variabilidad del número de inmersión en la cual se

Tabla 1. Conversión de color según guía Vita 3D-Master a cifra numérica.

\begin{tabular}{|c|c|c|c|c|c|c|c|c|c|c|c|c|c|}
\hline Color & Equ & Color & Equ & Color & Equ & Color & Equ & Color & Equ & Color & Equ & Color & Equ \\
\hline 1M1 & 1 & 2,5L1 & 16 & 2R1 & 31 & 3,5L1 & 46 & 3R1 & 61 & 4,5L1 & 76 & 4R1 & 91 \\
\hline 1M1,5 & 2 & L1,5 & 17 & 2R1,5 & 32 & $5 \mathrm{~L} 1,5$ & 47 & 3R1,5 & 62 & 1,5 & 77 & $\mathrm{R} 1,5$ & 92 \\
\hline 1M2 & 3 & $5 \mathrm{~L} 2$ & 18 & 2R2 & 33 & $3,5 \mathrm{~L} 2$ & 48 & 3R2 & 63 & $4,5 \mathrm{~L} 2$ & 78 & 4R2 & 93 \\
\hline $1 \mathrm{M} 2,5$ & 4 & 2,5L2,5 & 19 & 2R2,5 & 34 & 3,5L2,5 & 49 & 3R2,5 & 64 & $4,5 \mathrm{~L} 2,5$ & 79 & 4R2,5 & 94 \\
\hline $1 \mathrm{M} 3$ & 5 & 5L3 & 20 & 2R3 & 35 & $3,5 \mathrm{~L} 3$ & 50 & 3R3 & 65 & $4,5 \mathrm{~L} 3$ & 80 & 4R3 & 95 \\
\hline 1,5M1 & 6 & 2M1 & 21 & 2,5R1 & 36 & 3M1 & 51 & 3,5R1 & 66 & 4M1 & 81 & 4,5R1 & 96 \\
\hline 1,5M1,5 & 7 & 2M1,5 & 22 & 2,5R1,5 & 37 & $3 \mathrm{M} 1,5$ & 52 & 3,5R1,5 & 67 & $4 \mathrm{M} 1,5$ & 82 & 4,5R1,5 & 97 \\
\hline 1,5M2 & 8 & 2M2 & 23 & $2,5 \mathrm{R} 2$ & 38 & 3M2 & 53 & $3,5 \mathrm{R} 2$ & 68 & 4M2 & 83 & $4,5 \mathrm{R} 2$ & 98 \\
\hline 1,5M2,5 & 9 & $2 \mathrm{M} 2,5$ & 24 & 2,5R2,5 & 39 & $3 \mathrm{M} 2,5$ & 54 & $3,5 \mathrm{R} 2,5$ & 69 & $4 \mathrm{M} 2,5$ & 84 & 4,5R2,5 & 99 \\
\hline 1,5M3 & 10 & $2 \mathrm{M} 3$ & 25 & 2,5R3 & 40 & 3M3 & 55 & 3,5R3 & 70 & $4 \mathrm{M} 3$ & 85 & $4,5 \mathrm{R} 3$ & 100 \\
\hline 2L1 & 11 & 2,5M1 & 26 & 3L1 & 41 & 3,5M1 & 56 & 4L1 & 71 & 4,5M1 & 86 & 5M1 & 101 \\
\hline 2L1,5 & 12 & & 27 & 3L1 & 42 & & 57 & $4 \mathrm{~L} 1$ & 72 & $4,5 \mathrm{I}$ & 87 & $5 \mathrm{M} 1,5$ & 102 \\
\hline 2L2 & 13 & 2,5M2 & 28 & 3L2 & 43 & 3,5M2 & 58 & 4L2 & 73 & $4,5 \mathrm{M} 2$ & 88 & 5M2 & 103 \\
\hline 2L2,5 & 14 & 2,5M2,5 & 29 & 3L2,5 & 44 & 3,5M2,5 & 59 & $4 \mathrm{~L} 2,5$ & 74 & 4,5M2,5 & 89 & $5 \mathrm{M} 2,5$ & 104 \\
\hline 2L3 & 15 & 2,5M3 & 30 & 3L3 & 45 & 3,5M3 & 60 & 4L3 & 75 & $4,5 \mathrm{M} 3$ & 90 & $5 \mathrm{M} 3$ & 105 \\
\hline
\end{tabular}

Los puntos a analizados fueron los siguientes:

1.En qué número de inmersión en té ocurrió el primer cambio de coloración tanto en el GC como en el GNC (Tabla 2). Como el valor de $\mathrm{p}>0,05(0,207)$, es posible afirmar que los datos no son estadísticamente significativos, lo que indica que las medianas de los grupos GC y GNC están igualmente dispersas. Es decir, el primer cambio de color ocurrió en general durante la primera inmersión en té, y éste cambio ocurre a nivel del valor una de las tres esferas del color (Fig. 3).

2.En qué número de inmersión en té ocurrió el segundo cambio de coloración tanto en el GC como
Tabla 2. Prueba t para muestras independientes, primer cambio de color. Prueba de Levene para la igualdad de varianzas

Sig.

\begin{tabular}{|c|c|c|c|c|c|c|}
\hline Inmersión & $\mathrm{F}$ & sig & $\mathrm{t}$ & $\mathrm{Gl}$ & (bilateral) & DM \\
\hline $\begin{array}{l}\text { Se han asumido } \\
\text { varianzas iguales }\end{array}$ & 7.108 & ,011 & -1204 & 40 & ,203 &,- 571 \\
\hline $\begin{array}{c}\text { No se han } \\
\text { asumido varianzas } \\
\text { iguales }\end{array}$ & & & -1294 & 26,031 & ,207 &,- 571 \\
\hline
\end{tabular}

DM: diferencia de medias

Tabla 3. Prueba t para muestras independientes, segundo cambio de color. Prueba de Levene para la igualdad de varianzas

Sig.

\begin{tabular}{lcrcccc} 
Inmersión & $\mathrm{F}$ & $\mathrm{sig}$ & $\mathrm{t}$ & $\mathrm{gl}$ & (bilateral) & DM \\
\hline $\begin{array}{l}\text { Se han asumido } \\
\text { varianzas iguales }\end{array}$ & 12,889 &, 001 & $-2,329$ & 40 &, 025 & $-2,762$ \\
$\begin{array}{l}\text { No se han } \\
\text { asumido varianzas } \\
\text { iguales }\end{array}$ & & & $-2,329$ & 21,972 &, 207 & $-2,762$ \\
\hline
\end{tabular}

DM: diferencia de medias 


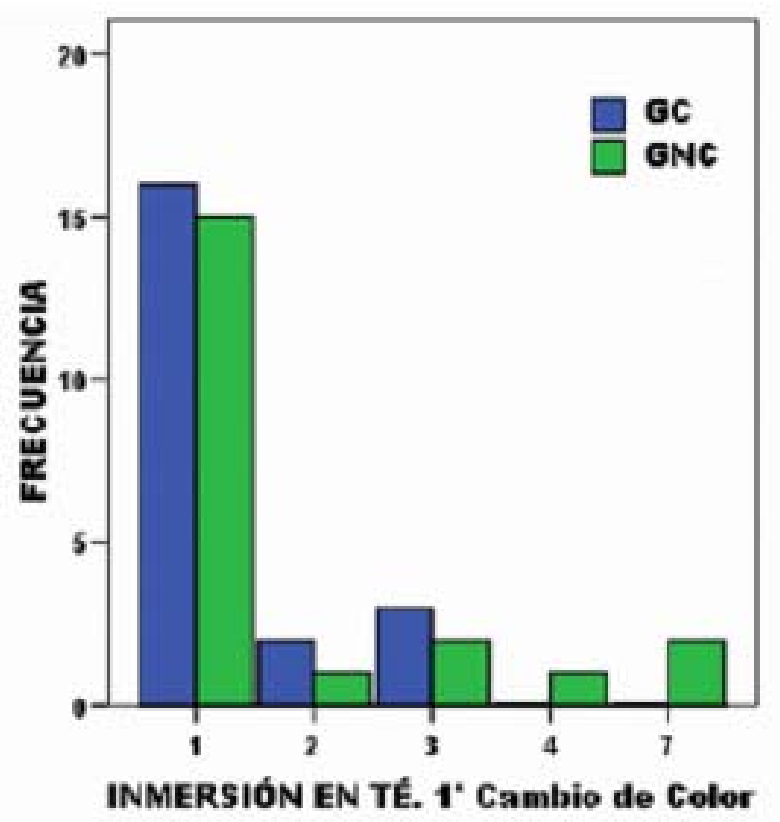

Fig. 3. Distribución del primer cambio de color según el número de inmersión en té en GC y GNC.

alcanza el color máximo por ambos grupos se encuentra igualmente disperso, es decir, la mediana de ambos grupos no difiere significativamente la una de la otra. Según lo que podemos ver en el diagrama de cajas (Fig.5), el GC experimentó su cambio de color máximo entre las 20 a 30 inmersiones en té, existiendo un percentil 75 que lo alcanzó de la inmersión 25 en adelante. El GNC, en cambio en su gran mayoría lo hace entre la décima a veinteava inmersión. Sin embargo, las medianas están muy próximas en ambos grupos por lo cual hace que las diferencia entre los GC y GNC no sean significativas.

4.Cuántos cambios de color totales fueron necesarios para que ambos grupos alcanzaran el máximo cambio de Color $(5 \mathrm{M} 3=105)$ (Tabla 5). Como el valor de $\mathrm{p}<0,05$ $(0,000)$, entonces es posible afirmar que los datos son estadísticamente significativos para decir que la mediana del GC es evidentemente distinta a la del GNC. Esto se evidencia al totales.

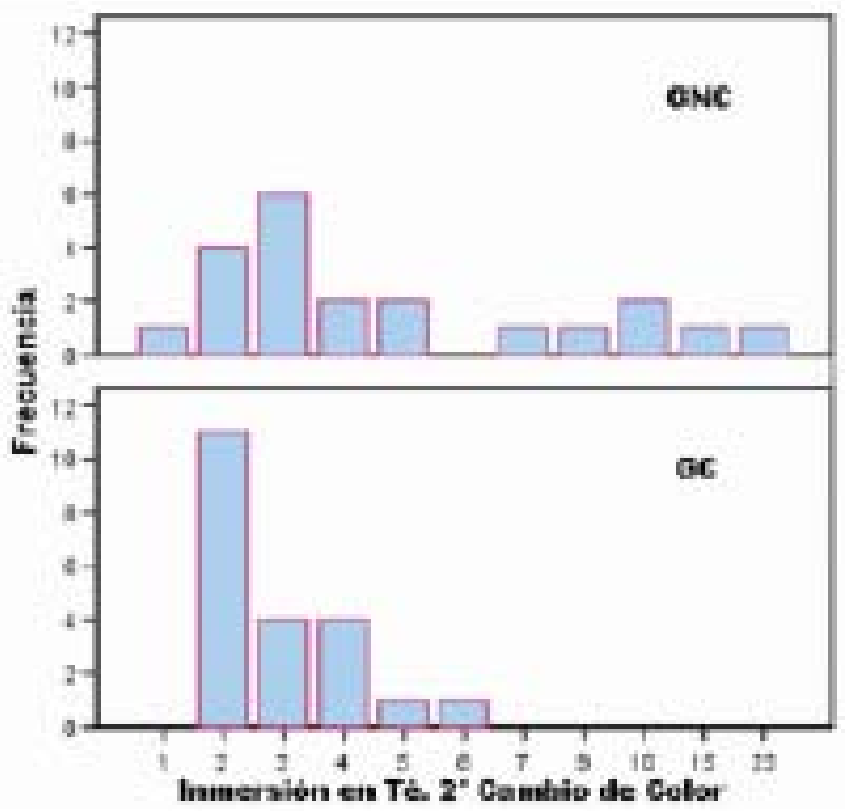

Fig. 4. Distribución del segundo cambio de color según el número de inmersión en té en GC y GNC. observar que el GC experimenta al menos seis cambios de color antes de llegar al máximo, no así el GNC que sólo presenta cuatro. Esto se muestra gráficamente en el diagrama de cajas (Fig.6).

5.Evaluar cómo es el cambio del color (rango o diferencia cuantitativa) experimentado por el
GC y GNC entre el primer y segundo cambio de color (Tabla $6)$. Al igual que en el caso anterior, el valor de $\mathrm{p}<0,05(0,001)$, entonces es posible afirmar que existe una diferencia significativa entre las medianas del GC y la del GNC. Esto se puede corroborar evaluando la diferencia entre el
Tabla 4. Prueba t para muestras independientes. Cambio máximo de color Prueba de Levene para la igualdad de varianzas

Sig.

\begin{tabular}{lccccrrr} 
Inmerscolorm & $\mathrm{F}$ & sig & $\mathrm{t}$ & $\mathrm{gl}$ & (bilateral) & DM \\
\hline $\begin{array}{l}\text { Se han asumido } \\
\text { varianzas iguales }\end{array}$ & 0,732 &, 397 & 1,114 & 40 & & ,272 & 4,524 \\
$\begin{array}{l}\text { No se han } \\
\begin{array}{l}\text { asumido varianzas } \\
\text { iguales }\end{array}\end{array}$ & & & 1,114 & 39,905 & \multirow{2}{*}{, 272} & 4,524 \\
\hline
\end{tabular}

DM: diferencia de medias

Tabla 5. Prueba t para muestras independientes. Número de cambios de color

\begin{tabular}{|c|c|c|c|c|c|c|}
\hline \multirow[b]{2}{*}{ Inmersión total } & \multicolumn{6}{|c|}{ Prueba de Levene para la igualdad de varianzas } \\
\hline & $\mathrm{F}$ & sig & $\mathrm{t}$ & Gl & $\begin{array}{c}\text { Sig. } \\
\text { (bilateral) }\end{array}$ & DM \\
\hline $\begin{array}{l}\text { Se han asumido } \\
\text { varianzas iguales }\end{array}$ & ,024 & 878 & 5,308 & 40 &, 000 & 2,190 \\
\hline $\begin{array}{l}\text { No se han } \\
\text { asumido varianzas } \\
\text { iguales }\end{array}$ & & & 5,308 & 39,802 & 000 & $-2,190$ \\
\hline
\end{tabular}

DM: diferencia de medias 


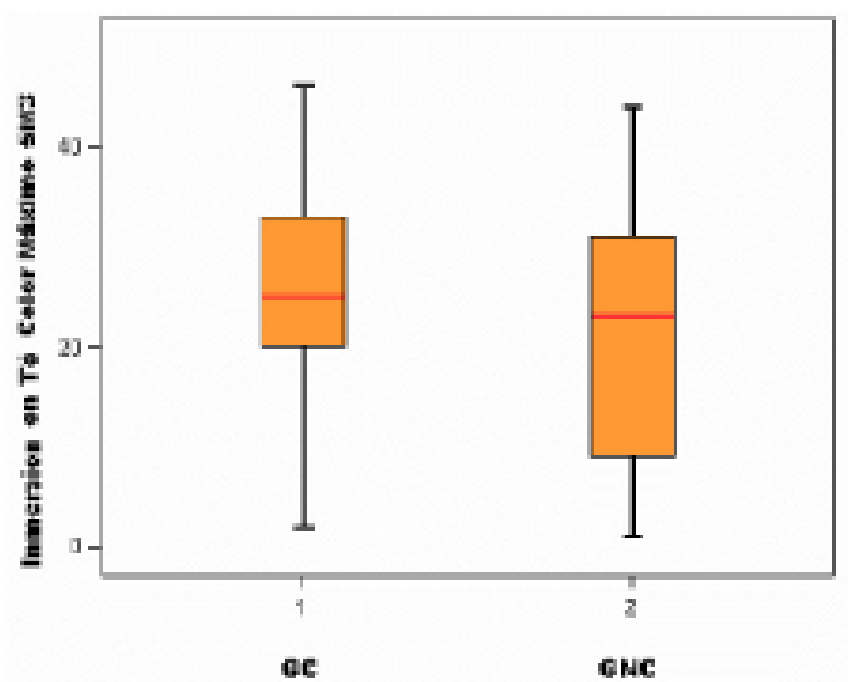

Fig. 5. Diagrama de cajas. Distribución en cuartiles del GC y GNC en el color máximo.

primer y segundo cambio de color experimentado por el GC, que es en general de 1 , esto ocurre a nivel de la característica del color denominada valor o claridad del color. No sucede lo mismo con el GNC donde la diferencia cuantitativa es menor de 0,5 ; también expresada en la característica valor del color.
6.Determinar si el color inicial del GC y del GNC tiene alguna relación en la cantidad de inmersiones en té necesarias para alcanzar el color máximo (5M3). Para realizar esta medición fue necesario utilizar un análisis de regresión lineal (Tabla 7). Como el valor de $p<0,05(0,000)$, es posible afirmar que frente al color
Tabla 6. Prueba t para muestras independientes. Diferencia de color del primer y segundo cambio Prueba de Levene para la igualdad de varianzas Sig.

\begin{tabular}{|c|c|c|c|c|c|c|}
\hline Dif.Color 1y2 & $\mathrm{F}$ & sig & $\mathrm{t}$ & gl & (bilateral) & DM \\
\hline Se han asumido & 42,96 & ,000 & 3,721 & 40 &, 001 & ,571 \\
\hline varianzas iguales & 9 & & & & & \\
\hline $\begin{array}{l}\text { No se han asumido } \\
\text { varianzas iguales }\end{array}$ & & & 3,721 & 24,208 & ,001 & , 571 \\
\hline
\end{tabular}

DM: diferencia de medias

Tabla 7. Análisis de regresión lineal. Influencia del color inicial en el número de inmersiones en té (color máximo).

Resumen del modelo

\begin{tabular}{cccccc}
\hline Modelo & R & R cuadrado & $\begin{array}{c}\text { R cuadrado } \\
\text { corregida }\end{array}$ & $\begin{array}{c}\text { Error tip. de la } \\
\text { estimación }\end{array}$ \\
\hline 1 &, $803(\mathrm{a})$ &, 645 &, 626 & 1,054 \\
\hline ANOVA(b) & & & & & \\
\hline Modelo & $\begin{array}{c}\text { Suma de } \\
\text { cuadrados }\end{array}$ & gl & $\begin{array}{c}\text { Media } \\
\text { cuadrática }\end{array}$ & F & Sig. \\
\hline 1 Regresión & 78,571 & 2 & 39,286 & 35,357 &, 000 \\
Residual & 43,333 & 39 & 1,111 & & \\
Total & 121,905 & 41 & & & \\
\hline
\end{tabular}

(a): Variables previctorias( Constante),grupo, colorinicial

(b): Variable dependiente: Inmersiontotal
Inicial hay diferencias estadísticamente significativas entre el GC y GNC, que indican una fuerte relación del color Inicial en el número total de inmersiones necesarias para alcanzar el color máximo. Con lo cual se concluye que un color inicial de menor valor requerirá un mayor número de inmersiones en té para alcanzar el color máximo (como es el caso del GC) y viceversa. (Fig.7).

7.Otras evidencias observadas en los resultados experimentales. No sólo existe un cambio en el valor en el color de las piezas dentarias en GC y en el GNC, sino que también en el Hue o tonalidad del color, de modo que siempre de un valor "L" o "R" la tendencia final es llegar a un nivel medio "M". Los cambios experimentados por el GNC en relación al paso de un color a otro, son más graduales y constantes que el GC, los cuales presentaron cambios más amplios de color (al inicio de las inmersiones en té) y más inestables en el tiempo, de modo que se evidenció regresiones de color y posteriormente avances, hasta alcanzar cierta estabilidad y progresión del color en las 

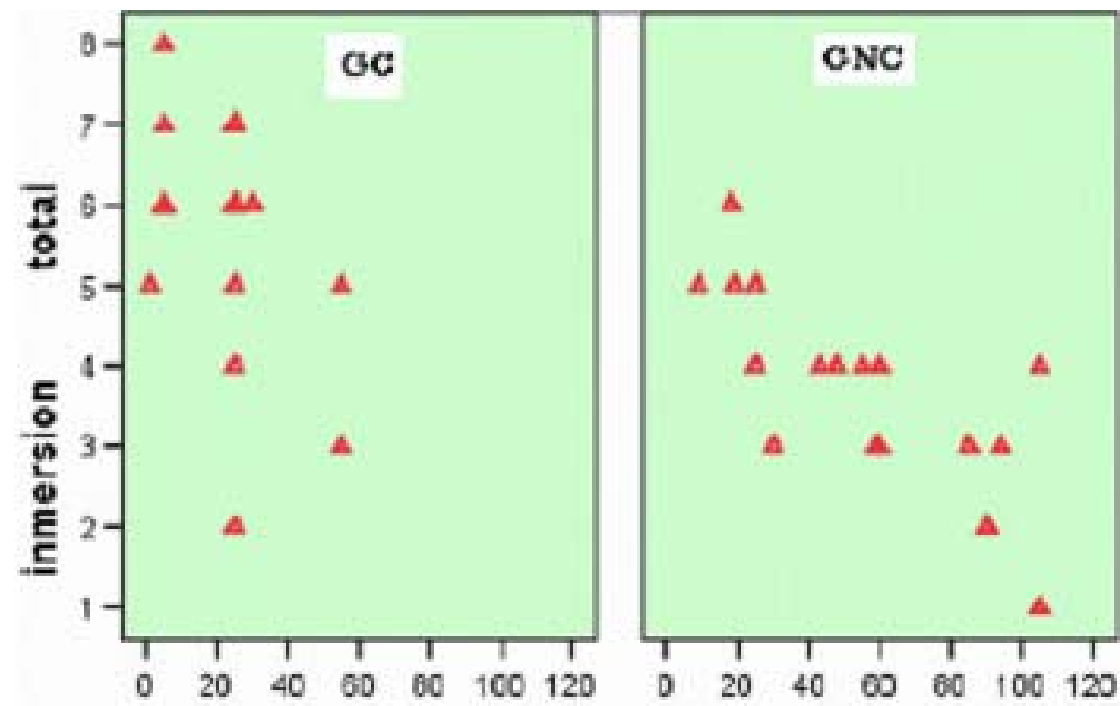

\section{color inicial}

Fig. 6. Gráfico de dispersión en relación al color inicial y número de inmersiones en GC y GNC.

inmersiones siguientes.

Relacionado con la situación anterior, el GC experimentó una marcada regresión del color a la $5^{\circ}$ inmersión en Té, la cual no se vuelve a repetir en las inmersiones siguientes. Esto no ocurre en el GNC.

\section{Discusión}

De acuerdo a los resultados obtenidos y frente a la hipótesis planteada, se puede afirmar que las piezas dentarias sometidas a clareamiento, si experimentan una regresión del color, la cual es de mayor magnitud que aquellas no tratadas. Esto es confirmado a través de las siguientes situaciones.

Tanto el GC como el GNC experimentaron mayoritariamente su primer cambio de color durante la primera inmersión en Té. Esto demuestra que el procedimiento de clareamiento no afecta, al menos inicialmente, la capacidad del esmalte dental para adquirir nuevas tinciones. Como lo comprobaron en un estudio In Vitro con dientes de bovinos, sometidos a clareamiento y posteriormente analizados microscópicamente, donde observaron que existen leves diferencias en la superficie del esmalte en relación al grupo no clareado, por lo cual se concluye que este tratamiento no alteraría significativamente la permeabilidad del esmalte, y por ende la interacción con nuevas tinciones (17).

Esta similitud entre los grupos analizados cambia en las inmersiones siguientes donde el GC sigue manifestando cambios en un menor número de inmersiones que el GNC. Esto sugiere que los cromógenos difunden rápidamente en el esmalte dental para saturar sitios disponibles dejados por la oxidación de los cromógenos eliminados. Como lo demostraron al analizar las decoloraciones generadas por Té en trozos de dientes humanos, viendo que éstas se generaban en mayor proporción en los primeros días de iniciado el contacto con este cromógeno, y que posteriormente esta decoloración se volvía más lenta (8).

En estudios similares se puede corroborar esta situación del GC, en los cuales se ha observado que el primer cambio de color experimentado por piezas bovinas clareadas ocurre en el primer día de inmersión en té, e incluso estas piezas manifestaron una regresión del color entre el 2 y 5 día de exposición. Además de no manifestar un evidente patrón de aumento en el color (18).

Si lo anterior lo extrapolamos a este estudio in vitro, se observará que a la quinta inmersión en té el GC experimentó una notoria regresión del color, y tampoco manifestó una constancia lineal en el aumento del color, más bien, se observó inestabilidad en la progresión de este. Esto quizás dice relación con el mecanismo por el cual se produce el efecto clareante, donde el agente permanece activo al menos cuatro horas después de la actividad inicial (alrededor del $60 \%)$ (19). Quienes al analizar la dinámica de degradación del agente clareador, confirmaron que la reacción ocurre con gran intensidad en un comienzo y luego decrece pausadamente (observado en dientes humanos extraídos). De manera que esta inestabilidad en la progresión del color en el GC puede ser dado por el efecto oxidativo residual presente en los túbulos dentinarios.

En este estudio in vitro se utilizaron piezas dentarias de bovinos las cuales presentan numerosas irregularidades en su superficie externa, la cual era diferente en cada espécimen, por lo cual esto puede influir en la generación de nuevas tinciones por té. Esto podría explicar en parte, que el rango o valor cuantitativo del cambio de color entre ambos grupos fuese mayor para el GC (a pesar que la selección de los especimenes de cada grupo fue al azar). En relación a esto, otra explicación del resultado obtenido podría ser, el efecto que ocasiona el agente clareante sobre la superficie 
del esmalte sobre todo a nivel de la textura superficial como descalcificación, y porosidades (20).

Sin embargo, hay investigaciones en las que se propone que dichos cambios pueden ser revertidos después de la remineralización producida por la saliva (8). Como en este estudio in vitro las piezas dentarias se mantuvieron en saliva artificial, no se puede afirmar si la mayor intensidad del cambio de color se deba a defectos de esmalte provocados por el agente clareante.

Como se mencionó en los resultados el cambio de color del GC y el GNC, no sólo ocurrió en el valor, sino que también en el Hue, de manera que piezas dentarias con un Hue de "L" o "R" a lo largo de las inmersiones se estabiliza a un "M". Esto puede ser explicado dado a la naturaleza del cromógeno del Té, compuesto principalmente de polifenoles como las teorubiginas y los teoflavinas, que son rojos y amarillos, respectivamente (21).

Otro factor a analizar es la temperatura de la solución de Té a la cual fueron inmersos tanto el GC y el GNC, la que presentó un rango entre los $64^{\circ} \mathrm{C}$ hasta los $44^{\circ} \mathrm{C}$ (periodo en el cual transcurren 10 minutos de inmersión), esto es para estandarizar la temperatura y controlar dicha variable, ya que hay estudios que señalan que a mayor temperatura de la infusión de té mayor es la intensidad o generación de decoloraciones. Así lo comprobaron al analizar el efecto de varias soluciones (té, café, agua destilada y clorhexidina) a distintas temperaturas, concluyendo que a medida que aumenta ésta la generación de pigmentaciones extrínsecas es mayor, especialmente la de solución de té (22).

Dentro de las implicancias clínicas que tiene este estudio es importante enfatizar a los pacientes, luego de realizado este procedimiento clínico, limitar o abstenerse de ingerir esta infusión para evitar tempranas pigmentaciones extrínsecas, en pro del mantenimiento del nuevo color adquirido. Por ello, se recomienda una "dieta blanca" las primeras dos semanas posterior al tratamiento de claramiento de manera que los cromógenos nuevos sean los que reemplacen a los eliminados (19).

Se sugiere complementar este estudio, analizando otras variables como por ejemplo, el efecto en el cambio de color de una profilaxis dental entre cada inmersión en té, determinando así si las pigmentaciones generadas son realmente extrínsecas, ya que hay estudios que sugieren que éstas pueden ser internalizadas.

\section{Conclusiones}

Según los datos obtenidos de este estudio in vitro, existe evidencia estadísticamente significativa, para afirmar que las piezas clareadas tienen un comportamiento totalmente distinto frente a un cromógeno (en este caso té) que las no clareadas, un color inicial más claro determina una regresión del color más rápida que las piezas no clareadas, con cambios de mayor intensidad y sin un patrón definido en la progresión de éste al ser sometido a este cromógeno.

El grupo de piezas dentarias no sometidas a clareamiento también presentan cambio de coloración en relación a su color inicial, existiendo diferencias significativas con el grupo clareado, de manera que el comportamiento del cambio de color de éstas piezas, presentaron una tendencia más definida adquiriéndose en forma paulatina.

Comparando ambos grupos se observa que es a partir del segundo cambio de color, en el cual el GC adquiere cambios mas intensos durante las primeras cinco inmersiones, el GNC lo hace posteriormente.

En relación a la diferencia cuantitativa del cambio de color entre el GC y el GNC, se determina que la intensidad del cambio es significativamente mayor en el GC que en el GNC.

\section{Referencias bibliográficas}

1. Joiner A. The bleaching of teeth: a review of the literature. J Dent. 2006; 34(7):412-9.

2. Berga Caballero A, Forner Navarro L, Amengual Lorenzo J. In vivo evaluation of the effects of $10 \%$ carbamide peroxide and $3.5 \%$ hydrogen peroxide on the enamel surface. Med Oral Patol Oral Cir Bucal. 2007; 12(5):E404-7.

3. Greenwall Linda. Técnicas de Blanqueamiento en Odontología Restauradora. Guía ilustrada., Editorial Ars Medica. $3^{\text {a Edición, }}$ 2002. España.

4. Touati, B.; et al.2003. Odontología Estética y Restauraciones Cerámicas. Boston: Masson. p: 40-59, 69-70, 87.

5. Goldstein Ronal E. Odontología Estética, volumen I, Principios, comunicación y métodos terapéuticos, editorial Ars medica, 2002. capitulo12, Pág.255-286.

6. Watts A, Addy M. Tooth discolouration and staining: a review of the literature. Br Dent J. 2001; 190(6):309-16.

7. Dahl JE, Pallesen U. Tooth bleaching--a critical review of the biological aspects. Crit Rev Oral Biol Med. 2003; 14(4):292-304.

8. Attin T, Wegehaupt F, Gries D, Wiegand $A$. The potential of 
deciduous and permanent bovine enamel as substitute for deciduous and permanent human enamel: Erosion-abrasion experiments. J Dent. 2007; 35(10):773-7.

9. Gracia Barbero Javier, Blanqueamiento dental interno (Cáp.15) en: Patología y Terapéutica dental, Editorial Síntesis, S.A. $2^{\circ}$ Edición, 2000. España.

10.Kashima-Tanaka M, Tsujimoto Y, Kawamoto K, Senda N, Ito K, Yamazaki M. Generation of free radicals and/or active oxygen by light or laser irradiation of hydrogen peroxide or sodium hypochlorite. J Endod. 2003; 29(2):141-3.

11. Camps J, de Franceschi H, Idir F, Roland C, About I. Timecourse diffusion of hydrogen peroxide through human dentin: clinical significance for young tooth internal bleaching. J Endod. 2007; 33(4):455-9.

12. Markovic L, Jordan RA, Lakota N, Gaengler P. Micromorphology of enamel surface after vital tooth bleaching. J Endod. 2007; 33(5):607-10.
13. Ghavamnasiri M, Bidar M, Rad AH, Namazikhah MS. The effect of 16 percent carbamide peroxide on enamel staining susceptibility. J Calif Dent Assoc. 2006; 34(11):873-6.

14. Heymann HO. Tooth whitening: facts and fallacies. Br Dent J. 2005; 198(8):514.

15. Ritter AV, Leonard RH Jr, St Georges AJ, Caplan DJ, Haywood VB. Safety and stability of nightguard vital bleaching: 9 to 12 years posttreatment. J Esthet Restor Dent. 2002; 14(5):275-85.

16. Cadenaro M, Breschi L, Antoniolli F, Mazzoni A, Di Lenarda R. Influence of whitening on the degree of conversion of dental adhesives on dentin. Eur J Oral Sci. 2006; 114(3):257-62.

17. Kwon YH, Huo MS, Kim KH, Kim SK, Kim YJ. Effects of hydrogen peroxide on the light reflectance and morphology of bovine enamel. J Oral Rehabil. 2002; 29(5):473-7.

18. Sulieman M, MacDonald E, Rees JS, Newcombe RG, Addy M. Tooth bleaching by different concentrations of carbamide peroxide and hydrogen peroxide whitening strips: an in vitro study. J Esthet Restor Dent. 2006; 18(2):93-100.

19.Leonard RH Jr. Efficacy, longevity, side effects, and patient perceptions of nightguard vital bleaching. Compend Contin Educ Dent. 1998; 19(8):766-70, 772, 774.

20.Justino LM, Tames DR, Demarco FF. In situ and in vitro effects of bleaching with carbamide peroxide on human enamel. Oper Dent. 2004; 29(2):219-25.

21. Sharif N, MacDonald E, Hughes J, Newcombe RG, Addy M. The chemical stain removal properties of 'whitening' toothpaste products: studies in vitro. $\mathrm{Br}$ Dent J. 2000; 188(11):620-4.

22. Pontefract H, Courtney M, Smith S, Newcombe RG, Addy M. Development of methods to enhance extrinsic tooth discoloration for comparison of toothpastes. 1. Studies in vitro. J Clin Periodontol. 2004; 31(1):16. 